The Clinical Journal of Pain Publish Ahead of Print

DOI:10.1097/AJP.0000000000000524

\title{
Investigating the fear avoidance model in people with whiplash: The
}

\section{association between fear of movement and in vivo activity}

Ashley Pedler, Ph. D. ${ }^{1,2}$

Steven J Kamper Ph. D. ${ }^{3}$

Annick Maujean Ph. D. ${ }^{1,2}$

Michele Sterling Ph. D. ${ }^{1,2}$

${ }^{1}$ Recover Injury Research Centre, Menzies Health Institute Queensland, Griffith University

${ }^{2}$ NHMRC Centre of Clinical Research Excellence in Road Traffic Injury Recovery, Griffith University

${ }^{3}$ The George Institute for Global Health, University of Sydney

Sources of funding

This project was supported by an Australian Research Council Discovery Grant, Canberra, ACT (ID: DP0772934). SK receives a fellowship funded by the National Health and Medical Research Council of Australia.

The authors declare no conflict of interest

Corresponding Author

Michele Sterling

Recover Injury Research Centre

Gold Coast Campus

Griffith University, QLD, 4222

Ph: +61 7488196862

Email: m.sterling@griffith.edu.au 


\begin{abstract}
Objectives: The aims of this study were to examine the relationship between fear of movement and activity levels in people with WAD over the first 3 months post injury, to determine the mediating effect of: fear of movement on the relationship between catastrophising and activity levels, and activity levels on the relationship between fear of movement and disability in patients with WAD.
\end{abstract}

Methods: Ambulatory monitoring of physical activity was conducted for a minimum of 8 waking hours on 2 consecutive days within 4 weeks post injury and at 12 weeks post injury for 103 (74 female) people with WAD. Time spent in upright postures (uptime) and time in motion were collected along with measures of pain, disability, pain catastrophising and fear of movement. The association of self-report outcome measures with uptime and time in motion were examined. Mediation analyses were performed to determine the mediating effect of: fear of movement on the relationship between catastrophising and activity, and activity levels on the relationship between fear of movement and disability.

Results: Fear of movement was significantly related to uptime but not time in motion. Mediation analyses showed that the relationship between fear of movement and disability was not mediated by activity levels, and that the relationship between catastrophising and activity levels was not mediated by fear of movement.

Discussion: Our data suggests measures of FAM are not related to general physical activity in people with WAD. Investigation of movements specific to the cervical spine and alternative explanatory models may be required.

Keywords: whiplash, fear avoidance, accelerometry, mediation 


\section{INTRODUCTION}

A significant proportion (30-60\%) of people who experience whiplash injury as the result of a motor vehicle collision (MVC) report ongoing pain and disability at long term follow up [1, 2]. The mechanisms underlying this chronic condition are yet to be fully elucidated, however the Fear Avoidance Model (FAM) has been proposed as one model which may explain the development of chronic disability in this population [3]. The FAM postulates that following an injury, movement or activity may result in increased pain. Catastrophic interpretation of this pain results in a maladaptive fear of movement initiating a cycle of avoidance, deconditioning and disability [4].

Studies examining the effect of fear of movement on pain and disability in patients with whiplash associated disorders (WAD) use self-report measures of both fear of movement and disability [5-7]. A more empirical measure to support the FAM as a process by which chronic disability is maintained in people with WAD would be to evaluate the effect of fear of movement on activity levels. Data from in vivo monitoring in patients with chronic WAD has shown that fear of pain, while related to self-reported pain, was not related to activity levels in the following hour[8]. In contrast, an association between self-reported fear of movement and in vivo activity using ambulatory monitoring has been reported in patients with chronic low back pain [9]. Higher levels of fear of movement or re-injury were associated with lower levels of activity [9].

Current clinical guidelines for the management of people with WAD advocate an active management approach with a focus on return to normal activities [10]. Identification of the factors which inhibit a return to usual activities are therefore of clinical importance in this population. In the framework of the FAM, fear of movement is one such factor. Recent work however has highlighted the lack of empirical data to support the structural assumptions of the FAM [11]. In particular insufficient evidence is available to confirm the sequential 
causative associations between catastrophic reactions to pain, fear of movement, avoidance of activity and disability $[12,13]$. Identification of the causative factors for reduced activity levels, and the influence of activity levels on disability would aid in development and application of appropriate management approach in people with WAD.

The aims of this study were 1) to examine the relationship between commonly used selfreport measures of fear of movement and in vivo activity levels in people with WAD over the first 3 months post injury 2) to determine if the relationship between catastrophising and activity levels is mediated by self-report measures of fear of movement and 3) to determine if the relationship between fear of movement and disability is mediated by activity levels as hypothesised by the FAM.

\section{MATERIALS AND METHODS}

\section{Participants}

Participants experiencing neck pain following an MVC were recruited from Brisbane and Sydney, Australia during the period 2007 - 2010. Participants were recruited from local hospital Emergency Departments, local medical and allied health practitioners and through general advertisement. To be included patients had to be classifiable as WAD grade I- III according to the Quebec Task Force system [14], aged between 18 and 65 years with a duration of symptoms of not more than 4 weeks. Exclusion criteria were a WAD injury classifiable as grade IV (fracture or dislocation) [14], concussion or other head injury as a result of the MVC, a previous history of a whiplash injury, a previous history of neck pain requiring treatment in the previous 12 months and the presence of any other medical condition which may have influenced the results of the study. Ethical approval was obtained from the human research ethics committees of the Human Research Ethics Committees of The University of Queensland and The University of Sydney. Participants provided written 
consent to participate prior to enrolment in the study. Participants were provided a small payment to compensate them for travel costs associated with attending assessments.

\section{Outcome measures}

\section{Questionnaires}

For sample description purposes demographic and injury-related information including age, gender, employment status, medication use and accident details were collected using a standardised questionnaire.

\section{Current pain intensity}

Average pain intensity over the preceding 24 hours were measured using a $10 \mathrm{~cm}$ visual analogue scale (VAS) with anchors of $0=$ no pain and $10=$ worst pain imaginable [15].

Neck Disability Index (NDI)

The Neck Disability Index (NDI) is a 10 item measure of neck pain related disability. Each item is rated on a 6 point scale with responses ranging from no disability ( 0$)$ to complete disability (5). An overall score is generated by summing the score for each item and multiplying by 2 . The NDI has been widely used in patients with WAD and is a validated, reliable and responsive measure [16].

Tampa Scale of Kinesiophobia (TSK)

The TSK is comprised of 17 items regarding beliefs about exercise and pain. Each item is scored on a scale of 1 to 4 with responses ranging from strongly disagree (1) to strongly agree (4). Items $4,8,12,14$, and 16 of the TSK have been shown to have poor psychometric performance [17]. A shortened version of the TSK excluding these items has been shown to have good psychometric performance $[17,18]$ and is recommended in preference to the full version. The 11 item version of the TSK is used in all analyses in this study. 
Pictorial Fear of Activities Scale - Cervical Spine (PFActS-C)

The PFActS-C is a pictorial based measure containing 47 items. Each item is made up of a photograph of a person performing activities which place biomechanical stresses on the cervical spine and a $10 \mathrm{~cm}$ visual analogue scale (VAS) with the anchors "No fear or anxiety" and "Extreme fear or anxiety". Using the VAS the fear or anxiety experienced while imagining performing the depicted activity is rated. For purposes of this study a computerised version of the PFActS-C was used which has been described in detail elsewhere [6]. To generate overall scores the mean rating from a sub-set of 19 items was used. This approach has been shown to valid and reliable in people with WAD [19].

\section{Pain Catastrophising}

The catastrophising scale of the coping strategy questionnaire (CSQ-C) is an established measure of catastrophic appraisals of pain. It consists of 6 catastrophic statements about pain (e.g. "I worry all the time about whether it will end."). The frequency of these thoughts are rated on a 7 point scale $(0=$ never do, $3=$ sometimes, $6=$ always $)$. Item scores are summed to give a total score between 0 and 42 with higher scores indicating a greater frequency of catastrophic thinking about pain.

\section{Ambulatory monitoring}

Activity levels were obtained using an ambulatory monitoring device (Lifeshirt, Vivometrics CA). The Lifeshirt utilises accelerometers sampling at $10 \mathrm{~Hz}$ to measure motion along the $\mathrm{x}$, $\mathrm{y}$ and $\mathrm{z}$ axes to provide data relating to posture and movement. Once the Lifeshirt had been fitted to the participant the accelerometers were calibrated to a known posture. Accelerometer data was used to identify the proportion of time spent in an upright posture (sitting or standing) and a percentage of time spent in motion. Accelerometer data was extracted using Vivologic software (Vivometrics, CA). Where two days of data were available the day with 
the longest period of recorded data was used for further analysis. Days with data recording periods less than 5 hours were excluded from further analysis.

\section{Uptime}

The Lifeshirt output includes a postural indicator which defines postural position as either upright (sitting/standing) or lying (prone/supine/left lateral/right lateral). Where the median postural indicator for a 1 minute epoch was upright this was defined as an upright minute. The percentage of uptime was calculated as the total number of uptime minutes divided by the total duration of the recording period in minutes.

\section{Time in motion (TIM)}

The Lifeshirt output includes a measure of activity representing the sum of accelerometer output across the $\mathrm{x}$ and $\mathrm{y}$ axes. Activity was assessed for every 1 minute epoch of data recorded. Participants were deemed to be active if the median activity level for a 1 minute epoch exceeded the threshold defined for slow walking defined by the manufacturer using the Vivologic software. This was deemed an active minute. A percentage of TIM was calculated using the number of active minutes divided by the duration of the recording period in minutes. We chose to use this measure rather than a measure of overall activity given that total duration of the recording period showed wide variation between participants potentially skewing measures of total activity time higher for participants with higher recording durations. Secondly we were interested in the tendency of participants to be active during the recording period rather than the intensity of any activities. The validity of accelerometry in the differentiation of inactivity versus activity is well established [20].

\section{Procedures}

Following agreement to participate, participants were scheduled for an initial assessment within 4 weeks of injury. Upon attendance at the research laboratory participants provided written consent and completed self-report questionnaires. A brief physical examination was 
then performed. Results of this examination are reported elsewhere and do not form part of this study [21]. Participants were then fitted with the Lifeshirt which was calibrated for accelerometer assessment of posture. Participants were shown how to fit and operate the Lifeshirt. Each participant wore the Lifeshirt for the rest of the day on which the assessment was performed. In order to ensure the recording of an entire day of activity assessments were scheduled as early as possible with recording starting before 10:15am. Participants were asked to wear the shirt until 8:30pm and to wear the Lifeshirt the following day for the same period with the aim of recording daily activities for 8 continuous hours on at least 1 of the monitoring days. Following the second day of recording the Lifeshirt was returned. Data was downloaded from the Lifeshirt and analysed using Vivologic software (Vivometrics, CA). All measures (questionnaires, physical assessment, ambulatory monitoring) were reassessed at 12 weeks post injury.

\section{Statistical analyses}

Effect of pain, catastrophising and fear of movement on activity

Separate random intercepts models with uptime and TIM as the dependent variables were constructed. Measures of average 24hr pain intensity (VAS), fear of movement (PFActS-C, TSK) and pain catastrophising (CSQ-C) were entered into each model as independent variables. Changes in the relationship between each independent variable and the dependent variable over time were assessed through inclusion of the interaction of each independent variable with time. Time was treated as a categorical value (baseline $=0,12$ weeks $=1$ ). Given that active minutes are most likely to occur during upright minutes, the influence of the independent variables of interest on TIM may be confounded by uptime. The association between TIM and uptime was examined. A moderate, statistically significant correlation $(\mathrm{r}=$ $0.31, \mathrm{p}<0.001)$ was observed. Uptime was therefore added as a covariate in the model with TIM as the dependent variable. A dichotomous categorical variable for time (baseline $=0,12$ 
weeks $=1$ ) was also included in each model. Age, gender and employment status have previously been shown to have an association with physical activity levels [9, 22]. A variable for employment status was created by grouping participants into those who reported being employed (whether full-time, part-time or self-employed) and those who reported being not employed, including those reporting home duties or retirement. Age, gender and employment were examined for correlation with both TIM and uptime. Weak ( $r<0.1$ for all analyses), non-significant ( $\mathrm{p}>0.5$ for all analyses) correlations were observed and these variables were therefore left out of the model. In order to reduce the effects of multicollinearity on longitudinal data analysis, scores for all independent variables were centred on the grand mean. The condition number of the variable set was also calculated to assess yiolation of the assumption of multicollinearity. The condition number is derived through performing a principle components analysis (PCA) on the dependent variables that will be entered into the model. A PCA solution for $k$ independent variables will provide individual eigenvalues for each of $k$ components. The condition number is calculated as the square root of the ratio of the largest eigenvalue to the smallest eigenvalue. Condition numbers $\geq 30$ are considered to indicate the presence of moderate multicollinearity with values $\geq 100$ indicating severe cases. All mixed model analyses were performed using IBM SPSS version 22 software (IBM, USA).

\section{Mediation Analyses}

Mediation analyses were conducted to test the hypothesis that the relationship between catastrophising and activity levels was mediated by fear of movement and that the relationship between fear of movement and disability was mediated by activity levels. These hypotheses were tested using both uptime and TIM as measures of activity and both TSK and PFActS-C as measures of fear of movement. Therefore 8 mediation analyses were performed to test: 1) that TSK mediates the relationship between CSQ-C and uptime; 2) that TSK 
mediates the relationship between CSQ-C and TIM; 3) that PFActS-C mediates the relationship between CSQ-C and uptime; 4) that PFActS-C mediates the relationship between CSQ-C and TIM; 5) that uptime mediates the relationship between TSK and NDI; 6) that uptime mediates the relationship between PFActS-C and NDI; 7) that TIM mediates the relationship between TSK and NDI; and 8) that TIM mediates the relationship between PFActS-C and NDI.

Guidelines for the design of experiments recommend that the independent variable, mediator and dependent variable are all collected across 3 time points. As we collected data at 2 time points, mediation analyses were conducted using the product of coefficients strategy which is appropriate for two-wave mediational studies [23]. This form of analysis is described in

Figure 1. Path $a$ is calculated as the effect of the independent variable at time 1 on the mediator at time 2 controlling for effect of the mediator at time 1. Path $b$ is calculated as the effect of the mediator at time 1 on the dependent variable at time 2 controlling for the effect of the dependent variable at time 1 . The mediation effect for the mediator can be calculated as the product $a b$. The $95 \%$ confidence intervals for the mediation effect were calculated using the distribution of the product method [23]. A variable is determined to have a mediating effect when the $95 \%$ confidence intervals do not include 0 . Prior to mediation analysis the distribution of the residuals for the regression analyses used to estimate paths $a$ and $b$ in Figure 1 were examined for normality. In order to prevent violation of the assumption of normal distribution of residuals, uptime, TIM and PFActS-C were all log transformed prior to mediation analyses using the following transformations:

$$
\begin{aligned}
& y_{\text {uptime }}=\log 10101 \text {-uptime } \\
& y_{\text {time in motion }}=\log 10(\text { timein motion }+1) \\
& y_{\text {PFActS }-C}=\log 10(\text { PFAct } S-C+1)
\end{aligned}
$$


All mediation analyses were performed using the Rmediation package [24] and the statistical software package R version 3.1.0 (The R Foundation for Statistical Computing, Austria). For all statistical analyses the significance level was set to $p<0.05$.

\section{RESULTS}

\section{Sample}

One hundred and three participants with acute WAD (74 [71.8\%] female, mean age $=39.7 \pm$ 13.9 years) were enrolled in the study. Seventy-two participants $(70.6 \%)$ were recruited via referral from local hospital Emergency Departments, 15 (14.7\%) through general advertisement and $16(15.5 \%)$ through referral from local medical and physiotherapy practitioners. At baseline assessment the mean 24hour pain intensity rating (10cm VAS) was $4.0 \pm 2.4$. Forty-nine $(47.6 \%)$ participants reported that they were involved in a compensation claim of who 19 had engaged the services of a solicitor. The majority of participants were employed or self-employed at baseline assessment $(74,71.8 \%)$ with $12(11.7 \%)$ unemployed and the rest of the sample either retired $(5,4.9 \%)$ or reporting home duties $(12,11.7 \%)$ as their main occupation. Demographic data for the sample are provided in Table 1. Due to technical faults accelerometer data was unavailable for 5 participants $(4.9 \%)$ at baseline assessment and 11 participants (10.7\%) at 12 week assessment. Seventeen participants $(16.5 \%)$ were lost to follow up at 12 weeks assessment. Data was therefore available for 98 participants at baseline assessment and 75 participants at 12 weeks follow up. Following exclusion of accelerometer data where the duration of monitoring was less than 5 hours, data for 88 participants was available at baseline assessment and 68 participants at 12 weeks follow up. Descriptive statistics for variables of interest are presented in Table 2. 


\section{Random intercepts models}

Correlations between dependent and independent variables included in the random intercept models are presented in Table 3.

\section{Uptime as dependent variable}

The condition number for the variables included in the model with uptime as the dependent variable was 2.8 indicating no violation of the assumption of multicollinearity. Examination of the distribution of the residuals for the random intercept model constructed with uptime as the dependent variable revealed a significant negative skew. Data for uptime was transformed using the formula:

$$
y=\log 10(101-\text { Uptime })
$$

The random intercept model was re-run using transformed uptime as the dependent variable. There was no significant effect of time indicating that uptime did not significantly change between baseline and 12 weeks follow up (Table 4). There was a significant effect for the association of TSK with uptime such that higher TSK scores were associated with greater uptime (Table 4). Estimates of fixed effects showed that a 1 unit increase in TSK above the sample mean was associated with a $6.7 \%(95 \% \mathrm{CI}=0.2 \%, 13.7 \%)$ increase in uptime. Though there was no significant main effect for PFActS-C on uptime, there was a significant interactive effect of PFActS-C with time (Table 4). There was a significantly stronger negative effect of PFActS-C on uptime at 12 weeks compared with baseline. At baseline a 1 unit increase in PFActS-C above the sample mean was associated with a $2.2 \%(95 \% \mathrm{CI}=-$ $14.4 \%, 22.2 \%$ ) decrease in uptime. At 12 weeks follow up a 1 unit increase in PFActS-C above the sample mean was associated with a $16.4 \%(95 \% \mathrm{CI}=-27.9 \%,-3.0 \%$.) decrease in uptime. Though significantly different between baseline and 12 weeks over time, the effect of PFActS-C on uptime was not significantly greater than 0 at either time point. 


\section{TIM as dependent variable}

The condition number for the variables included in the model with TIM as the dependent variable was 2.9 indicating no violation of the assumption of multicollinearity.

Results of the random intercept model constructed with TIM as the dependent variable revealed that controlling for uptime no independent variable has a statistically significant effect on TIM. The effect of pain on TIM approached but did not reach statistical significance

(Table 5). There was also no significant interactive effect of any independent variable with time (Table 5).

\section{Mediation analyses}

Mediating effect of fear of movement on the relationship between pain catastrophising and activity

For both TSK and PFActS-C, path $b$ (the effect of the mediator on the dependent variable, Figure 1) was non-significant (Table 6). The mediation effect (product of path $a$, and path $b$ ) of both PFActS-C and TSK on the relationship between CSQ-C and uptime was not statistically significant (Table 6). Therefore neither PFActS-C nor TSK had a significant mediating effect on the relationship between CSQ-C (pain catastrophising) and uptime. Neither PFActS-C nor TSK significantly mediated the relationship between CSQ-C (pain catastrophising) and TIM. Path $b$ was not significant for either TSK or PFActS-C (Table 6). The mediation effect was also non-significant for both PFActS-C and TSK (Table 6).

\section{Mediating effect of activity on the relationship between fear of movement and disability} In the analysis of the mediating effect of measures of movement on the relationship between fear of movement and disability neither measure of movement (uptime, time in motion) were found to significantly mediate this relationship. Neither path $a$, path $b$ nor the product of paths $a$ and $b$ were statistically significant in any of the mediation analyses (Table 7). 


\section{Post-Hoc Analysis}

Following our unexpected findings that higher TSK scores were associated with increased uptime in our random intercepts model we hypothesised that loading of scores on TSK items associated with the somatic focus subscale of the TSK may be responsible for this finding. These items are closely related to pain catastrophising. As a post-hoc investigation of this hypothesis the random intercept model for uptime was re-run with the activity avoidance and somatic focus subscales of the TSK included as independent variables. Somatic focus was significantly associated with uptime $(\mathrm{F}[1,116.1]=7.6, \mathrm{p}=0.007)$ such that higher somatic focus scores were associated with increased uptime. There was no significant effect of activity avoidance on uptime $(\mathrm{F}[1,117.0]=0.002, \mathrm{p}=0.97)$.

\section{DISCUSSION}

The FAM has been proposed as an explanatory model for the development of chronic disability in chronic pain conditions including WAD [3]. We examined the relationship of activity levels with measures of pain, pain catastrophising and fear of movement in people with WAD over the first 12 weeks post-injury. Our results suggest that fear of movement has a significant relationship with the proportion of time that people with whiplash spent in upright postures although the direction of this relationship was not in the expected direction. The relationship between pain catastrophising and in vivo activity levels was not mediated by measures of fear of movement, and the relationship between fear of movement and disability was not mediated by in vivo activity levels.

Empirical evidence has shown that active management approaches provide modest though usually transient gains in pain and disability in people with WAD [25, 26]. Current clinical guidelines for the management of people with WAD advocate an active management approach with the aim of a return to normal activities as soon as possible [10]. Clinicians 
therefore require an understanding of the factors which are limiting participation in usual activities. The FAM proposes that fear of movement leads to avoidance of movement leading to deconditioning which exacerbates pain and disability. The association between fear of movement and activity levels has rarely been tested using objective measure of physical activity. One study of patients with chronic WAD showed that fear of pain measured throughout the day using an electronic diary was predictive of next hour pain but had not significantly associated with activity levels in the proceeding hour [8]. Data obtained from other chronic pain populations has shown mixed results for the association between fear of movement and physical activity. In a sample of patients with chronic neck and back pain no significant correlation between TSK scores and aerobic conditioning or maximal strength measures was found [27], while TSK scores have been shown to be significantly associated with physical activity in a sample patients with chronic low back pain [9]. Our data suggest that fear of movement is associated with the proportion of time that people with WAD spend in upright postures but not with overall activity levels, the latter were more strongly associated with pain intensity.

We found an association between uptime and fear of movement, though the direction of this association varied depending on the measure of fear of movement used. Higher TSK scores were associated with more uptime irrespective of time post injury. TSK scores have been shown to represent multiple constructs and may be more closely related to catastrophising than fear of movement $[18,28]$. In a post-hoc analysis we found that higher scores on the somatic focus subscale of the TSK were significantly associated with increased uptime whereas scores on the activity avoidance subscale was not. This lends some support to the theory that the association between TSK scores and uptime reflects an association with some construct other than fear of movement. Future investigations should separate these subscales of the TSK. However given the post-hoc nature of this finding, replication is required. 
We did not collect data regarding the nature of employment in our sample. It is possible that a proportion of participants in our study were employed in occupations with high physical demands resulting in higher activity levels than would otherwise be observed. Concerns about fulfilling these demands and the potential for increased pain may have resulted in higher anxiety about pain and contributed to the association between TSK scores and uptime - though a similar association between TSK and activity levels may reasonably be expected in this case. Future work should consider stratification of the sample based on employment status as well as the physical demands of employment.

We observed no association between TIM and measures of fear of movement. When the proportion of time spent in upright postures was controlled for no variables had a significant effect on TIM, though the effect of pain intensity approached significance. These results may be in part explained by difficulty in maintaining static sitting postures in our sample.

Prolonged sitting is associated with neck pain [29] which may result in higher activity levels in these patients in comparison to patients with other chronic pain conditions. It is also of note that TIM is a measure of general activity and it is possible that such measures do not accurately reflect activity of the cervical spine. Cervical spine activity may have occurred during epochs categorised as inactive as overall accelerometer readings did not reach the required threshold. Cervical spine specific objective measures of activity may provide more accurate results however we are unaware such measures. Addressing fear of movement has shown promising results in people with WAD in one RCT [3]. The equivocal nature of our results suggest that further evidence for the influence of fear of movement on activity in people with WAD is required before interventions aimed at reducing fear of movement can be advocated for improving activity levels in this patient group. Additionally, it is possible 
that fear avoidant behaviours may be related to the intensity of activities and we did not measure this factor.

The FAM proposes that disability is maintained through a sequential and cyclical relationship between catastrophic interpretation of pain, fear of movement and avoidance of activity. This sequence of development has not been tested previously in people with WAD. We examined the mediating effect of measures of fear of movement on the relationship between early pain catastrophising and later activity levels, and the mediating effect of activity levels on the relationship between early fear of movement and later disability. We found no significant mediating effects regardless of the measure of fear of movement or activity used. Recent work has not supported a sequential development of the components of the fear avoidance model in other chronic pain populations $[12,13,30]$. However these studies have not examined the influence of fear on in vivo activity levels. Our data extend previous work through inclusion of such measures. Though we [6] and others [5, 31] have shown a significant relationship between TSK scores and disability in people with WAD, based on the current study, this relationship appears to be manifest through pathways other than those proposed in the FAM. The applicability of the proposed model to this patient population is contentious.

People with WAD are a heterogeneous group [32]. We did not limit our sample to only include those displaying elevated levels of psychological constructs related to the fear avoidance model. A recent clinical trial utilising a homogeneous sample of people with WAD and elevated PFActS-C scores showed a significant effect on disability of a CBT based therapy aimed at reducing fear of movement [3]. It is possible that the assumptions of the FAM hold in a subgroup of participants displaying high levels of fear. While the methods used here provide an accurate estimate of the mediating effect, three-wave studies would allow greater confidence in the results of mediation analysis [23]. In particular the 2-wave 
mediation analysis used here relies on the assumption of stationarity - that relationships between variables in the mediation model are consistent over time. Though we have shown that the effect of PFActS-C scores on uptime varies over time, stationarity cannot be formally tested with 2-wave data. In addition it is possible that our study design does not have the temporal resolution required to identify the sequential relationship between the cognitive changes proposed in the FAM. We have also only considered activity levels post injury. Differing results may be found if changes in activity relative to pre-injury levels are examined. Additionally it is possible that since the participants knew they were being monitored, this may have influenced actual activity levels. Given these limitations the results presented here should be interpreted with caution. A higher sampling frequency incorporating three or more waves of data collection may produce different results. More complex structural equation modelling could also be performed to include identification of the influence of symptoms of PTSD in people with WAD given the traumatic nature of the injury and the work done in other traumatic pain populations [33].

The FAM has been proposed as a mechanism for the development of chronic disability in people with WAD. We examined the relationship between factors of the FAM and in vivo activity levels in people with WAD over the first 12 weeks post-injury, and tested the sequential pathways between catastrophising, fear of movement, activity levels and disability proposed in the FAM. We found a significant association between fear of movement and time spent in non-upright postures however higher fear of movement was associated with longer time spent in upright postures. These results highlight that further work is required to understand the relationship between fear of activity and actual activity in people with WAD. We were unable to confirm the sequential development of catastrophising, fear of movement, activity levels and disability proposed by the FAM in people with WAD. Although the FAM may explain the development of chronic disability in a subgroup of people with WAD, 
alternative models should be considered. Continued rigorous examination of the FAM and other explanatory models for chronic pain are required.

\section{Figure Captions}

Figure 1: Method of estimating path a and $\mathrm{b}$ in two wave mediation analyses. $\mathrm{X}, \mathrm{Y}$ and $\mathrm{M}$ are the independent, dependent and mediator variables respectively. Subscript 1 and 2 denote baseline and 12 weeks data collection points. Path $a$ is the effect of $\mathrm{X}_{1}$ on $\mathrm{M}_{2}$ controlling for $\mathrm{M}_{1}$. Path $b$ is the effect of $\mathrm{M}_{1}$ on $\mathrm{Y}_{2}$ controlling for $\mathrm{Y}_{1}$. The product $a b$ provides an estimation of the mediating effect of $\mathrm{M}$ on the relationship of $\mathrm{X}$ and $\mathrm{Y}$. 


\section{References}

1. Sterling M, Hendrikz J and Kenardy J. Developmental trajectories of pain/disability and PTSD symptoms following whiplash injury. Pain 2010;150:22-28.

2. Carroll L, Holm L, Hogg-Johnson S, Cote P, Cassidy D, Haldeman S, Nordin M, Hurwitz

E, Carragee E, Van der Velde G, Peloso P and Guzman J. Course and Prognostic Factors for Neck Pain in Whiplash-Associated Disorders (WAD). Results of the Bone and Joint Decade 2000-2010 Task Force on Neck Pain and Its Associated Disorders. Spine 2008;33:583-592.

3. Robinson JP, Theodore BR, Dansie EJ, Wilson HD and Turk DC. The role of fear of movement in subacute whiplash-associated disorders grades I and II. Pain 2013;154:393-401.

4. Crombez G, Eccleston C, Van Damme S, Vlaeyen JW and Karoly P. Fear-avoidance model of chronic pain: the next generation. Clin J Pain 2012;28:475-83.

5. Vangronsveld K, Peters M, Goosens M and Vlaeyen J. The influence of fear of movement and pain catastrophizing on daily pain and disability in individuals with acute whiplash injury: a daily diary study. Pain 2008;139:449-457.

6. Pedler A and Sterling M. Assessing fear-avoidance beliefs in patients with whiplash associated disorders: a comparison of 2 measures. Clinical Journal of Pain 2011;27:502-507.

7. Buitenhuis J, Jaspers J and Fidler V. Can kinesiophobia predict the duration of neck symptoms in acute whiplash? . Clinical Journal of Pain 2006;22:272-277.

8. Sterling M and Chadwick B. Psychological Processes in Daily Life With Chronic Whiplash: Relations of post-traumatic stress symptoms and fear-of-pain to hourly pain and uptime. Clinical Journal of Pain 2010;26:573-582.

9. Alschuler KN, Hoodin F, Murphy SL, Rice J and Geisser ME. Factors contributing to physical activity in a chronic low back pain clinical sample: a comprehensive analysis using continuous ambulatory monitoring. Pain 2011;152:2521-7.

10. MAA Guidelines for the management of whiplash associated disorders. Sydney: Motor Accident Authority (NSW). www.maa.nsw.gov.au, 2014:16.

11. Wideman TH, Asmundson GG, Smeets RJ, Zautra AJ, Simmonds MJ, Sullivan MJ, Haythornthwaite JA and Edwards RR. Rethinking the fear avoidance model: toward a multidimensional framework of pain-related disability. Pain 2013;154:2262-5.

12. Wideman TH, Adams H and Sullivan MJ. A prospective sequential analysis of the fearavoidance model of pain. Pain 2009;145:45-51. 
13. Bergbom S, Boersma K and Linton SJ. Both early and late changes in psychological variables relate to treatment outcome for musculoskeletal pain patients at risk for disability. Behav Res Ther 2012;50:726-34.

14. Spitzer W, Skovron M, Salmi L, Cassidy J, Duranceau J, Suissa S and Zeiss E. Scientific Monograph of Quebec Task Force on Whiplash associated Disorders: redefining "Whiplash" and its management. Spine 1995;20:1-73.

15. Peters ML, Patijn J and Lame I. Pain assessment in younger and older pain patients: psychometric properties and patient preference of five commonly used measures of pain intensity. Pain medicine (Malden, Mass) 2007;8:601-10.

16. Vernon $\mathrm{H}$. The neck disability index: patient assessment and outcome monitoring in whiplash. Journal of Musculoskeletal Pain 1996;4:95-104.

17. Woby SR, Roach NK, Urmston M and Watson PJ. Psychometric properties of the TSK11: a shortened version of the Tampa Scale for Kinesiophobia. Pain 2005;117:137-44. 18. Roelofs J, Sluiter JK, Frings-Dresen MH, Goossens M, Thibault P, Boersma K and Vlaeyen JW. Fear of movement and (re)injury in chronic musculoskeletal pain: Evidence for an invariant two-factor model of the Tampa Scale for Kinesiophobia across pain diagnoses and Dutch, Swedish, and Canadian samples. Pain 2007;131:181-90.

19. Turk D, Robinson J, Sherman J, Burwinkle T and Swanson K. Assessing fear in patients with cervical pain: development and validation of the Pictorial Fear of Activity ScaleCervical (PFActS-C). Pain 2008;139:55-62.

20. Masse LC, Fuemmeler BF, Anderson CB, Matthews CE, Trost SG, Catellier DJ and Treuth M. Accelerometer data reduction: a comparison of four reduction algorithms on select outcome variables. Med Sci Sports Exerc 2005;37:S544-54.

21. Kamper S, Maher C, Hush J, Pedler A and Sterling M. Relationship between pressure pain thresholds and pain ratings in patients with whiplash associated disorders. Clinical Journal of Pain 2011;26:495-501.

22. Smuck M, Kao MC, Brar N, Martinez-Ith A, Choi J and Tomkins-Lane CC. Does physical activity influence the relationship between low back pain and obesity? Spine $J$ 2014;14:209-16.

23. Cole DA and Maxwell SE. Testing mediational models with longitudinal data: questions and tips in the use of structural equation modeling. Journal of abnormal psychology 2003;112:558-77.

24. Tofighi D and MacKinnon DP. RMediation: an R package for mediation analysis confidence intervals. Behavior research methods 2011;43:692-700. 
25. Michaleff Z, Maher C, Lin C, Rebbeck T, Connelly L, Jull G and Sterling M.

Comprehensive physiotherapy exercise program or advice alone for chronic whiplash (PROMISE): a pragmatic randomised controlled trial (ACTRN12609000825257). The Lancet 2014;384:133-141.

26. Teasell R, McClure J, Walton D, Pretty J, Salter K, Meyer M, Sequeira K and Death B. A research synthesis of therapeutic interventions for WAD: Part 4 - non invasive interventions for chronic WAD. Pain Research and Management 2010;15:313-322.

27. French DJ, France CR, Vigneau F, French JA and Evans RT. Fear of movement/(re)injury in chronic pain: a psychometric assessment of the original English version of the Tampa scale for kinesiophobia (TSK). Pain 2007;127:42-51.

28. Burwinkle T, Robinson J and Turk D. Fear of Movement: Factor Structure of the Tampa Scale of Kinesiophobia in Patients With Fibromyalgia Syndrome. Journal of Pain 2005;6:384-391.

29. Hallman DM, Gupta N, Mathiassen SE and Holtermann A. Association between objectively measured sitting time and neck-shoulder pain among blue-collar workers. Int Arch Occup Environ Health 2015;88:1031-42.

30. Lee H, Hubscher M, Moseley GL, Kamper SJ, Traeger AC, Mansell G and McAuley JH. How does pain lead to disability? A systematic review and meta-analysis of mediation studies in people with back and neck pain. Pain 2015;156:988-97.

31. de Jong JR, Vangronsveld K, Peters ML, Goossens ME, Onghena P, Bulte I and Vlaeyen JW. Reduction of pain-related fear and disability in post-traumatic neck pain: a replicated single-case experimental study of exposure in vivo. J Pain 2008;9:1123-34.

32. Pedler A and Sterling M. Patients with chronic whiplash can be subgrouped on the basis of symptoms of sensory hypersensitivity and posttraumatic stress. Pain 2013;154:1640-8. 33. Lopez-Martinez AE, Ramirez-Maestre C and Esteve R. An examination of the structural link between post-traumatic stress symptoms and chronic pain in the framework of fearavoidance models. Eur J Pain 2014;18:1129-38. 


\begin{tabular}{|c|c|c|}
\hline \multirow[t]{2}{*}{ Gender, n (\%) } & $\mathrm{F}$ & $74(71.8)$ \\
\hline & M & $29(28.2)$ \\
\hline \multicolumn{2}{|l|}{ Age (years), mean (SD) } & $39.7(13.9)$ \\
\hline \multicolumn{2}{|c|}{24 Hour Pain Intensity (10cm VAS), mean (SD) } & $4.0(2.4)$ \\
\hline \multirow[t]{5}{*}{ Compensation, n (\%) } & None & $51(49.5)$ \\
\hline & Third Party & $19(18.4)$ \\
\hline & Workers Compensation & $28(27.2)$ \\
\hline & Other & $2(1.9)$ \\
\hline & Missing & $3(2.9)$ \\
\hline \multirow[t]{2}{*}{ Engaged a Solicitor, n (\%) } & No & $82(79.6)$ \\
\hline & Yes & $19(18.4)$ \\
\hline \multirow[t]{5}{*}{ Employment Status, n (\%) } & Employed & $65(63.1)$ \\
\hline & Home Duties & $12(11.7)$ \\
\hline & Unemployed & $12(11.7)$ \\
\hline & Self Employed & $9(8.7)$ \\
\hline & Retired & $5(4.9)$ \\
\hline
\end{tabular}

\begin{tabular}{lll}
\hline Table 1: Sample demographic information & \\
\hline Variable & Baseline & 12 weeks \\
& Median (IQR) & Median (IQR) \\
\hline $\begin{array}{lll}\text { Recording duration* } \\
\text { (minutes) }\end{array}$ & $610.2(122.6)$ & $594.2(140.1)$ \\
Uptime & $78.8 \%(24.3 \%)$ & $81.4 \%(28.4 \%)$ \\
TIM & $9.9 \%(8.2 \%)$ & $10.7 \%(7.5 \%)$ \\
Pain & $4.0(4.0)$ & $2(2)$ \\
NDI & $33.8 \%(24 \%)$ & $22.8 \%(21.5 \%)$ \\
CSQ-C & $6.0(11)$ & $3.0(10)$ \\
TSK-11 & $26(9)$ & $22(12)$ \\
PFActS-C & $3.4(5.2)$ & $1.8(3.3)$ \\
\hline
\end{tabular}

Table 2: Descriptive statistics for variables at baseline and 12 week assessment displayed as Median (Interquartile range). TIM = Time in Motion, NDI = Neck Disability Index, CSQ-C = Coping Strategies Questionnaire - Catstrophising subscale, TSK = Tampa Scale of Kinesiophobia, PFActS-C = Pictorial Fear of Activity Scale - Cervical Spine.* Mean (SD). 


\begin{tabular}{ccccccc}
\hline Variable & Uptime & TIM & NDI & CSQ-C & TSK & PFActS-C \\
\hline TIM & $.315^{*}$ & & & & & \\
NDI & -.060 & $-.167^{*}$ & & & & \\
CSQ-C & -.131 & $-.219^{*}$ & $.619^{*}$ & & & \\
TSK & $.189^{*}$ & .140 & $.698^{*}$ & $.614^{*}$ & & \\
PFActS_C & .018 & .059 & $.627^{*}$ & $.472^{*}$ & $.506^{*}$ & \\
Pain & .005 & -.097 & $.688^{*}$ & $.440^{*}$ & $.523^{*}$ & $.427^{*}$
\end{tabular}

Table 3: Pearson correlation coefficients between independent and dependent variables included in the random intercept models. TIM $=$ Time in Motion, NDI = Neck Disabiltiy Index, CSQ-C $=$ Coping Strategies Questionnaire - Catstrophising subscale, TSK = Tampa Scale of Kinesiophobia, PFActS-C $=$ Pictorial Fear of Activity Scale - Cervical Spine. ${ }^{*}$ significant at $\mathrm{p} \leq 0.05$

\begin{tabular}{lcccc}
\hline Parameter & Numerator df & Denominator df & F & Sig. \\
\hline Intercept & 1 & 88.3 & 585.9 & $<.001$ \\
Time & 1 & 85.6 & 1.4 & 0.24 \\
Pain & 1 & 116.3 & 1.4 & 0.24 \\
TSK & 1 & 115.4 & 6.8 & 0.01 \\
PFActS-C & 1 & 116.1 & 2.9 & 0.09 \\
CSQ-C & 1 & 118.2 & 0.50 & 0.48 \\
Time * Pain & 1 & 109.9 & 0.57 & 0.45 \\
Time * TSK & 1 & 108.2 & 0.27 & 0.60 \\
Time * PFActS-C & 1 & 111.8 & 5.0 & 0.03 \\
Time * CSQ-C & 1 & 112.0 & 0.81 & 0.37 \\
\hline
\end{tabular}

Table 4: Results for tests of fixed effects for model with Uptime as the dependent variable. TSK = Tampa scale of kinesiophobia, PFActS-C = Pictorial Fear of Acitvity Scale - Cervical Spine, CSQ-C = Coping Strategies Questionnaire - Catastrophising subscale. 


\begin{tabular}{lcccc}
\hline Parameter & Numerator df & Denominator df & F & Sig. \\
\hline Intercept & 1 & 77.0 & 295.2 & $<.001$ \\
Time & 1 & 60.8 & $<0.001$ & 0.99 \\
Uptime & 1 & 92.6 & 5.4 & 0.02 \\
Pain & 1 & 108.8 & 3.8 & 0.054 \\
TSK & 1 & 111.3 & 0.18 & 0.67 \\
PFActS-C & 1 & 114.8 & 3.3 & 0.07 \\
CSQ-C & 1 & 107.9 & 2.2 & 0.14 \\
Time * Pain & 1 & 78.5 & 0.84 & 0.36 \\
Time * TSK & 1 & 69.6 & 1.5 & 0.23 \\
Time * PFActS-C & 1 & 81.6 & 0.03 & 0.86 \\
Time * CSQ-C & 1 & 71.2 & 0.19 & 0.66 \\
\hline
\end{tabular}

Table 5: Results for tests of fixed effects for model with Time in Motion as the dependent variable.

TSK = Tampa scale of kinesiophobia, PFActS-C = Pictorial Fear of Activity Scale - Cervical Spine, CSQ-C = Coping Strategies Questionnaire - Catastrophising subscale.

\begin{tabular}{|c|c|c|c|c|c|}
\hline Independent (X) & Mediator (M) & Dependent (Y) & B Path a & $\begin{array}{l}\text { B Path b } \\
\text { (p value) }\end{array}$ & $\begin{array}{l}\text { Mediation effect } \\
(95 \% \text { CI })\end{array}$ \\
\hline CSQ-C & TSK & Uptime & & $\begin{array}{c}-0.001 \\
(0.71) \\
0.01 \\
(0.20)\end{array}$ & $\begin{array}{l}-0.004,0.004 \\
-0.002,0.024\end{array}$ \\
\hline CSQ-C & PFActS-C & Time in Motion & $\begin{array}{c}0.02 \\
(<0.001) \\
-\end{array}$ & $\begin{array}{c}-0.004 \\
(0.66) \\
-0.03 \\
(0.21)\end{array}$ & $\begin{array}{l}-0.0004,0.0004 \\
-0.001,0.0004\end{array}$ \\
\hline
\end{tabular}

Table 6: Results of mediation analyses for the mediating effect of fear of movement on the relationship between catastrophising and activity levels. Path a is the effect of the independent variable at baseline on the mediator variable at 12 weeks when the value of the mediator at baseline is controlled for. Path $b$ is the effect of the mediator at baseline on the dependent variable at 12 weeks when the value of the dependent variable at baseline is controlled for. The mediation effect if the product of path a and path $b . \beta=$ standardised regression coefficient. 


\begin{tabular}{|c|c|c|c|c|c|}
\hline Independent (X) & Mediator (M) & $\begin{array}{l}\text { Dependent } \\
\text { (Y) }\end{array}$ & $\begin{array}{l}\beta \text { Path a } \\
\text { (p value) }\end{array}$ & $\begin{array}{l}\beta \text { Path b } \\
\text { (p value) }\end{array}$ & $\begin{array}{l}\text { Mediation effect } \\
(95 \% \mathrm{CI})\end{array}$ \\
\hline \multirow[t]{2}{*}{ TSK } & Time in motion & NDI & $\begin{array}{l}-0.001 \\
(0.71)\end{array}$ & $\begin{array}{l}-0.46 \\
(0.18)\end{array}$ & $-0.004,0.005$ \\
\hline & Uptime & & $\begin{array}{c}0.01 \\
(0.20)\end{array}$ & $\begin{array}{l}0.002 \\
(0.98)\end{array}$ & $-0.003,0.005$ \\
\hline PFActS-C & Time in motion & NDI & $\begin{array}{l}-0.004 \\
(0.66)\end{array}$ & $\begin{array}{l}-0.46 \\
(0.18)\end{array}$ & 0 \\
\hline & Uptime & & $\begin{array}{l}-0.03 \\
(0.21)\end{array}$ & $(008)$ & \\
\hline
\end{tabular}

Table 7: Results of mediation analyses for the mediating effect of activity levels on the relationship between fear of movement and disability. Path a is the effect of the independent variable at baseline on the mediator variable at 12 weeks when the value of the mediator at baseline is controlled for. Path $\mathrm{b}$ is the effect of the mediator at baseline on the dependent variable at 12 weeks when the value of the dependent variable at baseline is controlled for. The mediation effect if the product of path a and path b. $\beta=$ standardised regression coefficient. 
Figure
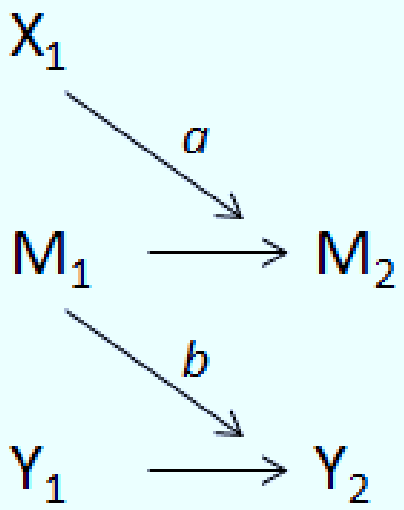

rs Kluwer Health, Inc. Unauthorized reproduction of 